\title{
Pour une histoire de la cartographie des territoires teko et wayãpi (Commune de Camopi, Guyane française)
}

A history of the Teko and Wayãpi (Commune of Camopi, French Guiana)

territories' cartography

Pierre Grenand, Françoise Grenand, Pierre Joubert et Damien Davy

\section{OpenEdition}

Journals

Édition électronique

URL : http://journals.openedition.org/ethnoecologie/3007

DOI : 10.4000/ethnoecologie.3007

ISSN : 2267-2419

Éditeur

Laboratoire Eco-anthropologie et Ethnobiologie

Référence électronique

Pierre Grenand, Françoise Grenand, Pierre Joubert et Damien Davy, « Pour une histoire de la cartographie des territoires teko et wayãpi (Commune de Camopi, Guyane française) », Revue d'ethnoécologie [En ligne], 11 | 2017, mis en ligne le 03 juillet 2017, consulté le 19 avril 2019. URL : http://journals.openedition.org/ethnoecologie/3007 ; DOI : 10.4000/ethnoecologie.3007

Ce document a été généré automatiquement le 19 avril 2019

\section{cc)}

Revue d'ethnoécologie est mis à disposition selon les termes de la licence Creative Commons Attribution - Pas d'Utilisation Commerciale - Pas de Modification 4.0 International. 


\title{
Pour une histoire de la cartographie des territoires teko et wayãpi (Commune de Camopi, Guyane française)
}

\author{
A history of the Teko and Wayãpi (Commune of Camopi, French Guiana) \\ territories' cartography
}

Pierre Grenand, Françoise Grenand, Pierre Joubert et Damien Davy

\section{Introduction}

1 Les Wayãpi et les Teko (anciennement nommés Oyampi et Emérillon) sont deux peuples amérindiens de la grande famille tupi-guarani, largement répandue au Brésil. Leurs langues présentent de sensibles différences phonétiques, lexicales et grammaticales. En 2016 la population amérindienne de la commune de Camopi avoisine les 1700 personnes, toutes locutrices de l'une ou l'autre de ces deux langues, parfois des deux et l'intercompréhension se généralise. 
Figure 1 : Carte de la commune de Camopi - Toponymie wayãpi et teko de l'Oyapock et de la Camopi

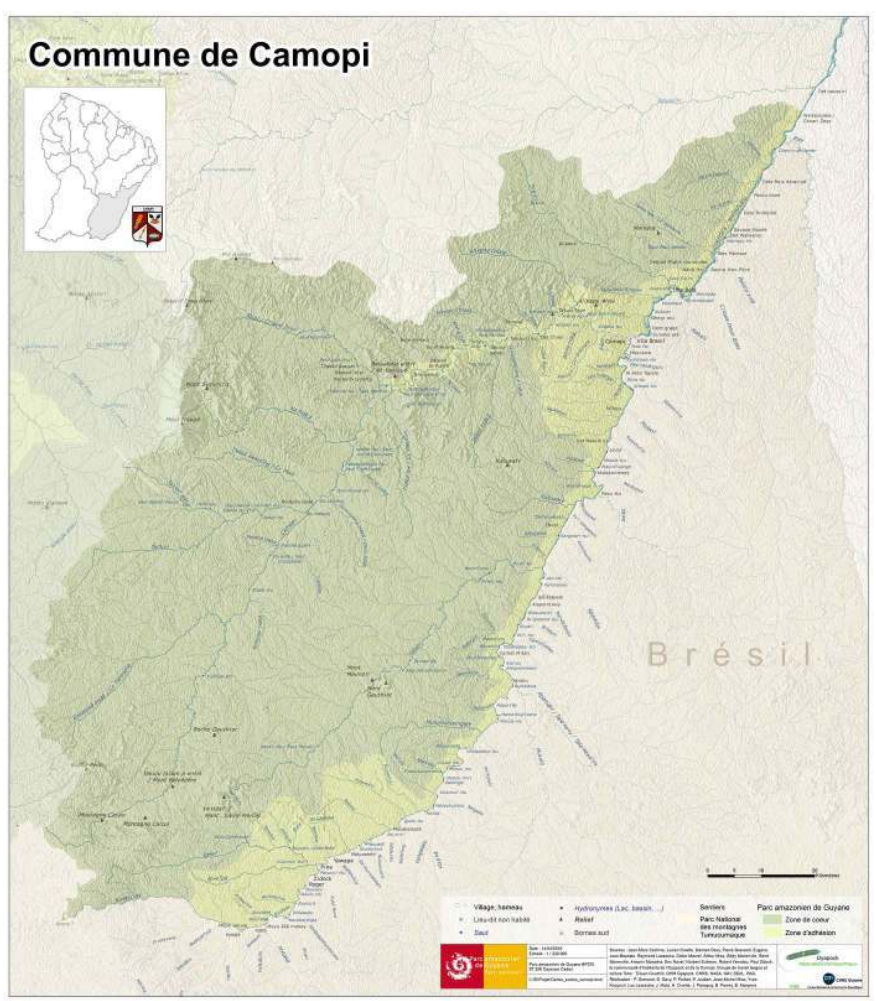

2 Le territoire des Wayãpi de Guyane et des Teko s'est construit, depuis deux siècles, autour du fleuve Oyapock et de ses affluents, dont la rivière Camopi. Dans le cas des Teko ce territoire s'étend cependant aux formateurs orientaux du Maroni. Aux XVII ${ }^{\mathrm{e}}$ et $\mathrm{XVIII}^{\mathrm{e}}$ siècles les bassins des deux cours d'eau étaient habités par des populations de langues tupi-guarani et caribe, dont certaines se sont fondues tant avec les Teko et les Wayãpi qu'avec les Wayana du haut Maroni.

3 Aux XVII et XIX ${ }^{e}$ siècles, les Teko, formant l'une des composantes des peuples de langues tupi-guarani évoqués, ont habité diverses parties de la Guyane centrale avant de se répartir plus au sud au xxe siècle entre un groupe du Tampok (affluent du Maroni) et un groupe de la Camopi. Les Wayãpi, originaires du bas Xingu, se sont installés entre le Jari et l'Araguari (Amapá, Brésil) au XVIII ${ }^{\mathrm{e}}$ siècle puis sont entrés en Guyane française par les sources de l'Oyapock au début du XIX ${ }^{\mathrm{e}}$ siècle, occupant progressivement son bassin jusqu'en aval du confluent de la Camopi.

4 L'ensemble du bassin de l'Oyapock jusqu'à Canari-Zozo et celui de la Camopi jusqu'au Mont Belvédère furent de 1800 à 1980 le domaine exclusif des peuples Wayãpi et Teko, à l'exception d'orpailleurs artisanaux créoles dans la moyenne Camopi. La frontière francobrésilienne, devenue bien réelle à partir des années 1980, est venue restreindre les activités sur la rive droite du fleuve depuis la création au Brésil du Parque Nacional Montanhas do Tumucumaque; à cela s'ajoute le regain de l'orpaillage illégal depuis la fin des années 1990.

5 La décroissance démographique et la concentration de l'habitat sont des périodes révolues. Aujourd'hui, ce vaste territoire reste parcouru par les habitants, qui y puisent une bonne partie des ressources indispensables à leur mode de vie. Ils sont les seuls à 
connaître par le détail tous les sauts et leurs passes, les criques, les bancs de sable, les reliefs, les layons de chasse, les zones de collecte et de pêche, les villages anciens... Cette connaissance longue et intime de leur territoire s'exprime dans le très grand nombre de toponymes dont il est maillé.

La mise en cartes de ces toponymes est une pratique ancienne dans la région avec une participation variable des populations locales qui va plutôt en s'accentuant au cours du temps. Nous présentons donc ces différentes cartographies de la région de l'Oyapock en insistant particulièrement sur les expériences menées dans le long terme par les deux premiers auteurs de cet article. Plus récemment, les habitants de la commune de Camopi ont participé de façon particulièrement active et avec l'appui des autorités locales, à l'élaboration de cartes qui synthétisent et complètent les travaux précédents. Il est important de souligner que ces cartes les plus récentes (et désormais utilisées dans les écoles) sont le résultat d'une recherche participative menée, entre autres partenaires (Davy, Trisch et Grenand 2012), en collaboration avec le Parc Amazonien de Guyane créé en 2007 et dans lequel vivent désormais les Teko et les Wayãpi de Camopi.

\section{Histoire du présent travail}

7 Les toponymes apparaissant sur les cartes anciennes et modernes de la Guyane correspondent à l'accumulation de noms recueillis tout au long de son histoire coloniale. De nombreux noms ont disparu, d'autres ont subsisté comme partout ailleurs dans le monde. Pour la région de l'Oyapock nous disposons de nombreuses cartes anciennes aujourd'hui déposées à la Bibliothèque nationale (Paris) ou aux Archives d'Outremer (Aixen-Provence). À de rares exceptions, les personnes qui traçaient la plus grande partie de ces cartes ne parlaient ni les langues amérindiennes ni plus tard les langues maronnes ou le créole. Néanmoins plusieurs d'entres elles sont de la main d'hommes de terrain (Duvillard, Audiffrédy, Mentelle... au XVIII ${ }^{\mathrm{e}}$ siècle, Coudreau au XIX ${ }^{\mathrm{e}}$ ) et montrent qu'une riche toponymie indigène recouvrait l'ensemble de la région. En effet, les Européens qui pénétrèrent ces territoires, s'ils n'étaient pas aveugles, étaient pour le moins muets, dépendant totalement de la parole amérindienne pour nommer l'inconnu.

Ces cartographes seront relayés officiellement au $\mathrm{xx}^{\mathrm{e}}$ siècle par l'Institut géographique national (IGN) dont la tâche est de produire et d'amender des cartes de l'espace géographique et politique de la France. Pour le sud de la Guyane, cette cartographie méthodique commence en 1947, avec le travail pionnier de l'un de ses géographes les plus émérites, l'ingénieur Jean Hurault, qui se prit de passion pour le pays et ses habitants. En une quinzaine d'années, s'appuyant tant sur les photos aériennes que les relevés de terrain, il réussit avec son équipe à cartographier définitivement la Guyane. Si la toponymie du littoral s'est révélée être à peu près stable depuis le XIX ${ }^{e}$ siècle, il fallut encore et toujours faire appel au savoir des habitants du sud de la Guyane, Boni, Créoles et Amérindiens, pour nommer les lieux de cette partie du pays. Les cartes produites à des échelles très diverses entre 1950 et 1965 demeurent le matériel géographique de base pour toute recherche entreprise en Guyane.

9 Parallèlement, depuis une quarantaine d'années, la France se veut soucieuse de rendre compte de la diversité nationale partout où la langue d'oïl (à la base du français standard) n'est pas la langue historique. C'est le cas pour la Provence, le Languedoc, la Corse, le Pays Basque, la Bretagne, les Antilles... La notion de patrimoine, parallèlement à celle 
d'environnement, est devenue un terme essentiel des débats politiques nationaux et régionaux. Et la valorisation du patrimoine est aujourd'hui inséparable de celle des langues régionales, apparaissant dès lors sous leur forme écrite sur les cartes des territoires, entre autres supports.

Tournons-nous vers la Guyane et plus particulièrement l'Oyapock. Ce qui suit est une histoire dans l'histoire de ces cartes et concerne essentiellement Pierre et Françoise Grenand. Nous résumons en quelques lignes ce qui pourrait être un roman. Fin 1968 et durant l'essentiel de l'année 1969, ils effectuent un voyage aventureux les amenant à refaire une partie des voyages des explorateurs Crevaux et Coudreau, remontant le Maroni jusqu'à sa source, franchissant le partage des eaux, descendant le Mapahoni et une partie du Jari, remontant son affluent le Kouc, franchissant à nouveau le partage des eaux pour parvenir à Trois-Sauts, village récemment fondé par les Wayãpi. Au cours de ce voyage, ils furent successivement accompagnés de Noirs Marrons Ndjuka, de Wayana, d'Apalai, de Wayãpi du Sud et de Wayãpi du Nord, mais aussi de chercheurs d'or et de tantalite brésiliens. L'important ici est de retenir que les deux voyageurs disposaient d'une carte inédite faite d'éléments disparates tentant de localiser au mieux les communautés, ce que les cartes officielles ne fournissaient que partiellement. Cette errance permit aux deux auteurs seniors du présent article d'entrer de plain pied dans la géographie amérindienne. En particulier, ils découvrirent les talents de cartographes des Wayana, des Apalai et in fine des Wayãpi : 1- Ils traçaient des cartes sur le sable dont la particularité était qu'elles partaient toutes du lieu où parle la personne ; 2- Ils lisaient sans effort notre carte sur les mêmes bases ; 3- Ils traduisaient les localisations des cours d'eau, des montagnes et des villages par une gestuelle spatiale extrêmement précise et imagée.

11 La géographie amérindienne n'allait plus les «lâcher » jusqu'à ce jour, qu'il s'agisse de rendre compte de l'histoire, de migrations, de terroirs ou encore de délimiter des zones de droits d'usage. En bref, nous balayâmes un large champ allant de la recherche "pure " à son application militante dédiée. Précisons que pour l'essentiel de la période allant de la fin des années 1960 au début des années 1990 la notion de "recherche participative " n'est en aucun cas présente dans le discours des acteurs du monde dit « indigéniste ».

12 Puisque cartographie participative il y a, il nous est essentiel de préciser qu'elle fut avant tout affaire de conjonction. Celle-ci résulte de facteurs que nous résumerons sans fard. 1émergence d'une classe d'âge politisée et francophone tant chez les Teko que les Wayãpi ; 2- recherche scientifique en sciences sociales accordant une place plus importante à l'ontologie ; 3- existence d'un Parc national fournissant de nouvelles possibilités à la fois de dialogue et surtout de transferts technologiques.

13 Cette conjonction s'est traduite par une participation de l'ensemble des communautés teko et wayãpi de l'Oyapock et de la Camopi. Le rôle de l'ancien maire de la commune de Camopi, René Monerville, a été central puisqu'il a emporté l'adhésion de tous. Pour lui, Wayãpi et Teko participant d'un même passé, cartographier ce territoire devenait le symbole et l'aboutissement d'une histoire fédératrice s'ouvrant sur un futur commun.

14 Dans ce sens, les relevés toponymiques de la rivière Camopi sont révélateurs. Les données sur cette région étaient très fragmentaires. Quelques éléments avaient été relevés par Éric Navet (1985) dans le cadre de ces travaux historiques. De plus, la carte IGN présentait de nombreux noms créoles, issus de la période de l'orpaillage allant des années 1880 à 1940. C'est dans la Camopi que l'expression «recherche participative» a pris toute sa 
valeur puisqu'il s'est agi de recueillir en un temps relativement restreint un maximum de données et de les soumettre à un maximum de personnes.

Figure 2 : Occupation amérindienne de l'Oyapock et du Camopi du XVIII siècle à nos jours
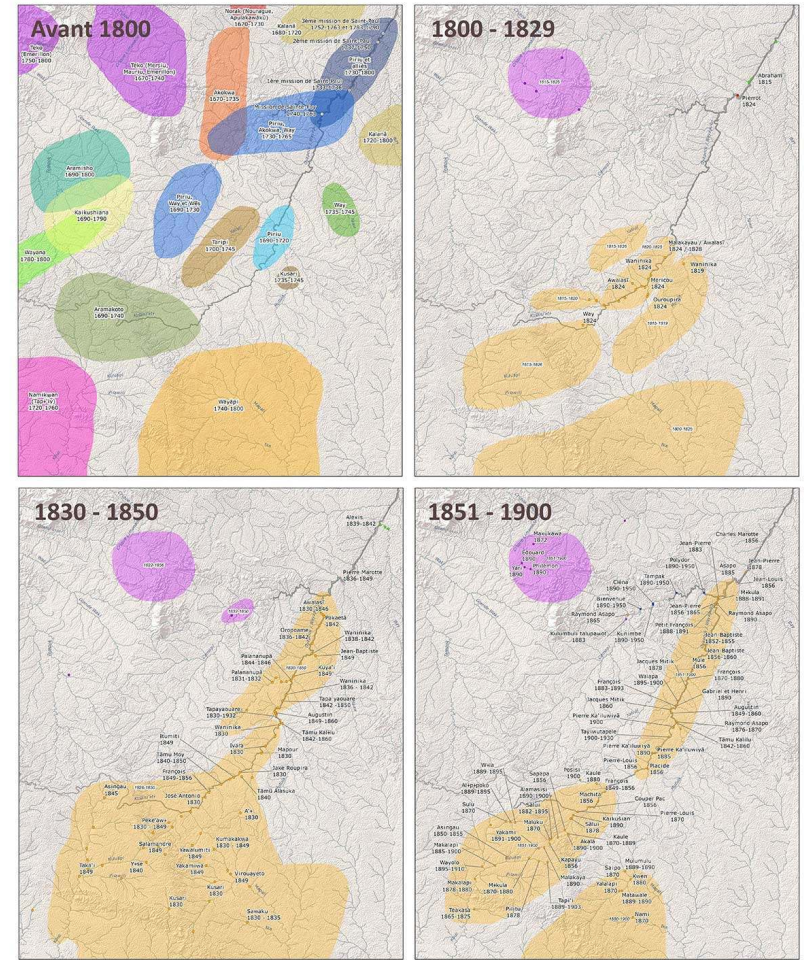

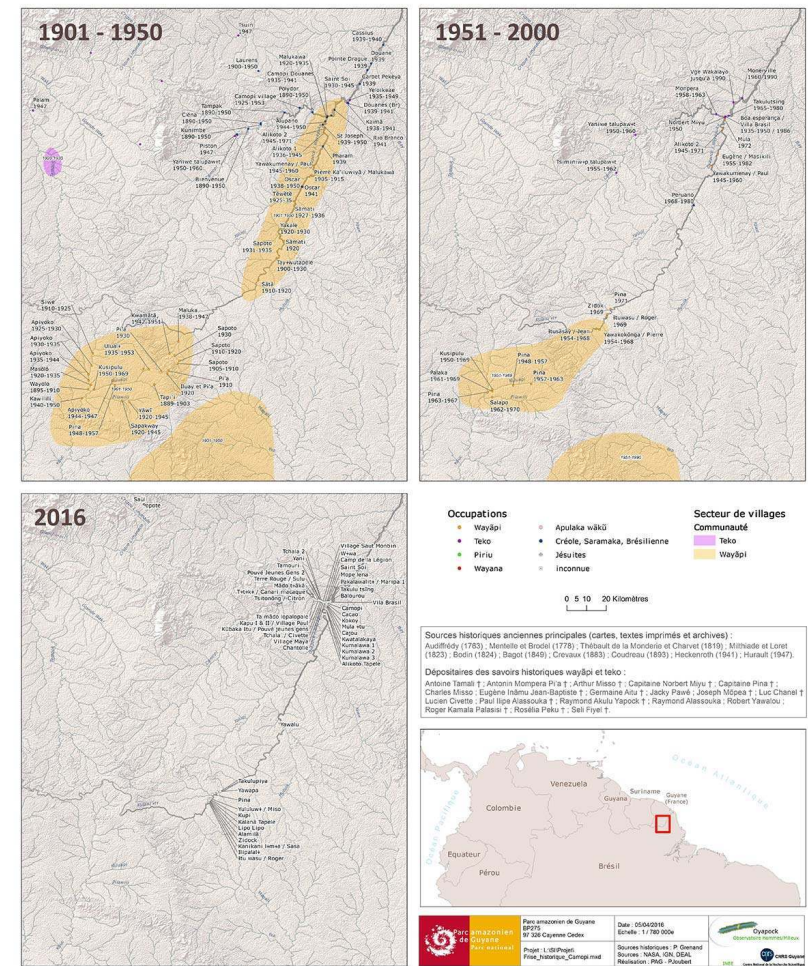

Sources historiques anciennes principales (cartes, textes imprimés et archives) : Audiffrédy (1763) ; Mentelle et Brodel (1778) ; Thébault de la Monderie et Charvet (1819); Milthiade et Loret (1823) ; Bodin (1824) ; Bagot (1849) ; Crevaux (1883) ; Coudreau (1893) ; Heckenroth (1941) ; Hurault (1947) Dépositaires des savoirs historiques wayãpi et teko :

Antoine Tamalit ; Antonin Mompera Pi'at ; Arthur Missot ; Capitaine Norbert Miyut ; Capitaine Pinat ; Charles Misso ; Eugène Inãmu Jean-Baptistet ; Germaine Aitut ; Jacky Pawé ; Joseph Mõpea† ; Luc Chanelt ; Lucien Civette ; Paul Ilipe Alassoukat; Raymond Akulu Yapockt ; Raymond Alassouka ; Robert Yawalou ; Roger Kamala Palasisit ; Rosélia Pekũt ; Seli Fiyel†

15 Il n'en reste pas moins que l'ensemble des cartes produites témoigne du temps long et représente, concrètement, un effort conduit sur moins de cinq ans. Cet effort a été permis par l'émergence des technologies modernes. Travailler avec Pierre Joubert, cartographe du Parc amazonien de Guyane, sur des cartes issues d'observations par satellites fut une révolution. Nous avons pu préciser non seulement des données issues de nos carnets, mais d'autres remontant parfois au $\mathrm{xVIII}^{\mathrm{e}}$ siècle. Il convient de préciser que cet enthousiasme fut largement partagé et surtout totalement réapproprié par l'ensemble des Amérindiens participant à l'aventure. Chaque peuple a probablement une vision particulière de sa géographie, mais pour ce qui est des Wayãpi et des Teko, la nécessité de nommer l'espace connu nous est apparue comme une activité intellectuelle particulièrement vive. Notre amie Dominique Gallois, anthropologue de l'Université de São Paulo (USP), nous a plusieurs fois évoqué l'enthousiasme avec lequel les Wayãpi du Sud avaient participé massivement à la délimitation de leur Terre indigène, là encore la toponymie étant au cœur de l'affaire.

Résumons-nous ! En capitalisant des relevés effectués depuis bientôt cinquante ans et en effectuant, de 2009 à 2011, un relevé systématique au GPS, nous avons pu dresser des cartes de la commune de Camopi comportant près de 600 toponymes différents transcrits dans les langues wayãpi et teko. Divers toponymes créoles ont été maintenus car ils font désormais partie de l'histoire de la région. Précisons que de nombreux Amérindiens sont d'excellents locuteurs du créole de Guyane. En 2012 et 2013, plusieurs réunions 
participatives se sont déroulées à Cayenne et dans les communautés de Camopi, permettant de vérifier l'ensemble des données. Les contributeurs à cet effort de cartographie participative sont présentés dans la légende des cartes. Le financement des opérations 2009-2011 et la spatialisation de ces données ont été obtenus grâce à une convention de recherche entre le Parc amazonien de Guyane et le CNRS (OHM Oyapock). Une entreprise partageant le même esprit a été conduite par Marie Fleury avec les Wayana et les Boni du Maroni (Fleury et al. 2016). Un travail identique est désormais possible pour la région du bas Oyapock dans un contexte de multilinguisme (créole, palikur, portugais du Brésil) après les révisions effectuées par Pauline Laval (2016).

Parallèlement aux cartes toponymiques, un jeu de cartes historiques a pu être dressé. La première, qui compile la localisation des ethnies anciennes au XVIII siècle, correspond à un souhait des Amérindiens, en particulier celui de M. René Monnerville. Les autres cartes suivent sur deux siècles par tranche de 25 à 50 ans la destinée des peuples Teko et Wayãpi et des autres habitants de la région. Les données historiques sont issues de divers travaux ethnologiques ici compilés (Grenand 1982, Navet 1985, Grenand et al. 2000, Davy et al. 2012). Ces cartes concernent les Teko à partir de la période où ils commencent à peupler le bassin de la Camopi, et les Wayãpi après leur entrée en Guyane. Pour la période la plus ancienne, elles s'appuient sur les archives et les documents imprimés. Les noms de village - noms de chefs ou de lieux-dits - sont reproduits dans leur orthographe française originale. Pour les périodes après 1830 , ces documents ont pu être recoupés et largement complétés par les détenteurs de savoir wayãpi et teko dont les noms figurent sur la carte. Tous les toponymes sont alors transcrits dans l'orthographe actuelle des langues wayãpi et teko.

\section{Remarques sur l'écriture des langues wayãpi et teko}

Les langues wayãpi et teko disposent désormais d'une écriture basée sur l'Alphabet phonétique international (API). La diffusion de ces écritures a répondu essentiellement à des demandes réitérées des jeunes Amérindiens qui considéraient que l'écriture du français ne permettait pas de restituer divers phonèmes de leurs langues dont le célèbre « i barré » (i) qui, à lui seul, est devenu un symbole de cette question et de surcroît un sujet de plaisanteries au sein des trois ethnies amérindiennes du sud de la Guyane. Les transcriptions des toponymes amérindiens depuis le XVII ${ }^{\mathrm{e}}$ siècle illustrent sur le temps long cette dure réalité. Initiées par P. et F. Grenand pour le wayãpi et par Éric Navet poursuivi de façon déterminante par Didier Maurel pour le teko, l'adoption de cette écriture s'est largement faite en marge de l'Éducation nationale (F. Grenand 2009 ; Maurel 2009).

Pour permettre aux non-locuteurs de ces deux langues de les prononcer correctement, voici quelques indications des sons (phonèmes) qui leur sont propres.

Le phonème $[\mathbf{u}]$ est prononcé comme le ou du français «loup ». Le phonème [i] $]$, absent des langues européennes, mais présent dans de nombreuses langues amérindiennes, est une voyelle prononcée entre le $\mathbf{i}$ et $\mathbf{u}$ du français. Le phonème [e] est prononcé é comme

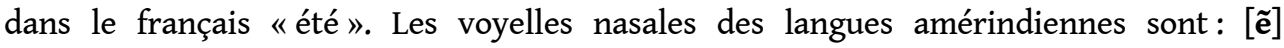

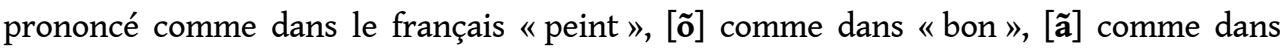

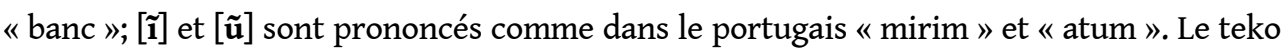
écrit $[\mathbf{b}]$ se prononce $\mathbf{m b}$, comme dans de nombreuses langues africaines. Le son [0̈] se 
prononce eu comme dans le français "peur». Les consonnes finales présentes en teko comme $\mathbf{k}, \mathbf{m}, \mathbf{n}, \mathbf{p}, \mathbf{t}$ sont toutes prononcées.

\section{Pourquoi et comment nommer?}

21 La toponymie d'un territoire reflète l'importance de l'appropriation de son environnement par une société. Aussi n'est-il pas étonnant que la rivière Camopi, territoire teko, possède plus de 150 toponymes : sauts, criques, courants, anciens villages, lieux-dits souvenirs d'événements historiques ou anecdotiques. Car exploiter son territoire, c'est savoir le parcourir et le connaître. Le connaître, c'est tout simplement le nommer. Voilà le seul moyen de l'inscrire dans la durée, de rendre compte de son histoire, de permettre aux assises du passé de vivifier le présent. Dans le contexte actuel où il existe de nombreux enjeux liés à la terre et à la langue, revendiquer la richesse toponymique de son territoire représente tout simplement un véritable acte de vie politique et culturelle.

22 Le cas des Wayãpi et des Teko contredit totalement la notion de savoir immémorial. I s'agit bien plutôt d'un savoir cumulatif, puisant tout à la fois dans celui des autres (ceux qui les ont précédés jadis), que dans celui des ancêtres récents. Dans le cas tout à fait remarquable des Teko, sont inclus dans leur vision de l'espace géographique leurs partenaires saramaka et créoles. Contrairement à ce que nous aurions pu déduire de certains propos plus ou moins ethnicistes de personnes vivant durement la réalité contemporaine, tous les habitants traversant le $\mathrm{xx}^{\mathrm{e}}$ siècle sont présents dans la vallée du Camopi, de la tante Mado qui tomba dans la vase d'un dégrad à la grand-mère Choua qui faillit être noyée par son père tant elle pleurait, sans oublier ce caïman vorace de Yaniwe qui dévora tant de piroguiers dans les temps anciens. Géographie immédiate, géographie éternelle... La fascination du sujet réside donc dans l'accumulation, sans pour autant qu'il $y$ ait confusion entre les acteurs.

23 Au-delà de ce cadre cumulatif, la toponymie que nous avons cartographiée fut strictement énoncée par les habitants amérindiens de la commune de Camopi. Elle reflète leur représentation de l'environnement à travers une terminologie stable. Les Wayãpi et les Teko définissent trois grandes catégories permettant de rendre compte de leur espace de vie : 1)- le village (taa), 2)- les zones défrichées périphériques comme les abattis (koo/ ko) ou les anciens abattis (koke/tapelet) et enfin 3)- les zones de parcours quotidiens ou épisodiques au sein de la forêt (ka'a), celle-ci constituant une mosaïque de milieux également nommés. Ce sont des zones emboîtées, de la plus humanisée (le village) à la plus sauvage (la forêt), pouvant être considérées comme des nébuleuses d'aires de parcours, reliées entre elles par les chemins des hommes (pe) et les cours d'eau naturels ( lì/tiãaã). Précisons que la notion de limite ou encore de frontière nationale n'existait pas, il y a encore quelques décennies, pour les peuples amérindiens de Guyane, comme d'ailleurs pour l'ensemble des peuples indigènes d'Amazonie.

On remarquera assez rapidement que les eaux vives, fleuve, rivières et ruisseaux, sont beaucoup plus riches de toponymes que la grande forêt. On peut penser que, pour cette partie de la Guyane tout du moins, l'absence presque absolue de larges horizons ou de points de vue remarquables a participé à la structuration d'une perception linéaire du territoire. Ainsi, les layons de chasse sont très souvent nommés par la crique ou le criquot desquels ils sont voisins. 

connaissance intime de ce territoire par ses habitants. Sans surprise, ce sont les régions les plus périphériques aux territoires parcourus qui sont les moins couvertes de noms. Cette toponymie en lignes et en points remarquables (et non pas en zones ou en aires) figure bien sûr les moyens de se repérer spatialement. On notera dans cet esprit que la région la moins pourvue en toponymes du cours de l'Oyapock correspond à un linéaire d'une quarantaine de kilomètres allant du confluent de la Notaye aux premiers grands sauts en aval, d'où les Amérindiens sont aujourd'hui absents et où les Créoles ne se sont jamais implantés.

Mais la toponymie des Wayãpi et des Teko va bien au delà de sa fonction géographique évidente: elle dessine toute une géographie mythique, culturelle ou plus simplement événementielle du territoire, et témoigne, au moins pour l'essentiel, d'une histoire antérieure à leur arrivée, dont les détenteurs de savoir wayãpi et teko ont conscience d'être les dépositaires pour les temps présents et les relais pour les temps futurs.

\section{Les villages}

Les toponymes de village sont souvent construits sur le nom d'un arbre. Ainsi sur la Camopi, il existe les villages teko nommés Tsitonõng (Citron), Titikö (Canari-macaque), sur le moyen Oyapock les villages Maripa, Mombin... Dans le pays wayãpi du haut Oyapock, on rencontrera le village Yululuwi (Sous l'arbre bois-ara, Parkia pendula)... Cette façon de nommer les villages se trouvant sur les très anciennes cartes, on peut penser qu'elle existait déjà chez les peuples de la région aujourd'hui éteints. Elle correspond d'une façon générale à l'importance de nommer le monde à partir d'un point référentiel.

Il n'est pas rare que de vieux toponymes désignant des sauts ou des lieux-dits et dont l'étymologie reste obscure, aient été réappropriés, comme c'est le cas de Masikili ou de Alikoto qui apparaissent sur la Carte de Audiffrédy (1763), donc antérieurement à l'arrivée des Wayãpi dans le bassin de l'Oyapock. Ces noms renvoient invariablement y compris jusqu'à nos jours au monde invisible.

Les villages peuvent également être nommés du nom de leurs fondateurs, manière très ancienne de leur rendre hommage. C'est le cas des villages Roger, Zidock ou encore Civette qui portent le nom des chefs qui les ont fondés, Roger Kamala, Zidock Yawapini, Roger Civette. Ça ne les empêche pas d'être aussi nommés respectivement ftu wasu (Trois Sauts), Pasisiwi (Sous l'arbre goupi) ou Tsala itu (Saut du grand-père Tsala). Le créole, dont nous avons situé la place historique, reste une langue tout autant maternelle que véhiculaire sur l'Oyapock, et a lui aussi laissé son contingent de toponymes (Dégrad SaintPierre sur la Camopi). Au final, on peut se retrouver avec des lieux nommés dans ces trois langues, chaque nom pouvant avoir, ou non, la même signification.

\section{Les cours d'eau et les sauts}

Les cours d'eau principaux du bassin de l'Oyapock étaient déjà nommés dès la pénétration des premiers Européens. Ainsi l'Oyapock et la Camopi apparaissent dès la fin du Xvi siècle pour le premier et à la fin du XviI ${ }^{\mathrm{e}}$ pour le second. Leurs noms seront utilisés avec des variantes proches jusqu'à nos jours. Notaye (ttãy), Sikini, Yawe, Yaroupi (Yalupi) et Moutoula (Mitũla) sont autant d'exemples qui apparaissent dès le milieu du XVIII ${ }^{e}$ siècle.

Revue d'ethnoécologie, 11 | 2017 
Tous ces noms sont considérés par les Wayãpi et les Teko comme antérieurs à leur arrivée; ce qui ne les empêchent pas de donner à certains une étymologie. Ainsi Itãy signifie « eau pimentée».

Les cours d'eau secondaires, linéaires structurant véritablement leur territoire, ont été majoritairement renommés dans les années 1830, avec l'avancée progressive des Teko et des Wayãpi. Ils s'appuient tous sur les suffixes -li en wayãpi et tiããa en teko signifiant rivière, affluent ou crique selon l'appellation guyanaise, comme Tuyukumuli (Crique des feuilles pourries), Pekeali (Crique de l'arbre pekea), Kulumulili (Crique des bambous), Mãndo tiãkã (Crique de Mado), Alat a tiãkã (Crique de l'ara rouge)...

Les sauts (itu dans les deux langues) sont construits de la même façon; on trouve ainsi le long de l'Oyapock ou de la Camopi des noms comme: Uluku itu (Saut roucou), Dzawapitãng a itu (Saut du tigre rouge = Saut du puma), Paila itu (Saut de l'arc)...

\section{Des noms liés à la nature (végétaux, animaux)}

Environ $50 \%$ des noms évoqués ci-dessus font directement référence au monde naturel, qu'il soit végétal ou animal, montrant l'importance du milieu pour les Teko et les Wayãpi, mais surtout la connaissance fine qu'ils en ont. Rappelons simplement que ces derniers nomment 1200 taxons végétaux, utilisent 272 plantes médicinales, ainsi que plus de 200 plantes à usage technique dont 57 pour la seule activité de vannerie (Grenand et al. 2004, Davy 2007).

Néanmoins la référence à la flore et à la faune relève de circonstances variables. Pour la flore, il s'agit le plus souvent de points de repère remarquables dans le paysage. Les nombreux Kulũbuli itu/Kulumuli itu sont des sauts marqués par des peuplements de bambous à crochets (Guadua latifolia), faciles à visualiser. D'autres combinent flore et faune comme sur la Camopi, le lieu-dit Bodjuhu lapat, (Roseaux à flèche de l'anaconda).

\section{Des lieux légendaires...}

Une grande partie de la toponymie tire également son origine d'épisodes légendaires incluant en particulier les antres d'animaux fabuleux. Ces termes, stables sur le temps long puisqu'on les trouve déjà dans les textes anciens, constituent un maillage structurant du territoire. Ils indiquent par dessus tout que les peuples de la région, au delà de leur mobilité du moment, partagent une même conception immanente du monde. Ainsi les Teko connaissent sur la Camopi un lieu nommé Bodjuju namitsila (Anaconda cornu), créature extraordinaire et dangereuse, déjà indiqué par le voyageur Patris en 1761. Une île rocheuse de cette même rivière est appelée Wakula dzawat (Engouleventjaguar), monstre qui dévore ceux qui le nomment. De même, l'Oyapock est parsemé de lieux où se sont déroulés des épisodes mythiques importants, comme par exemple Willapolaytawe, l'« Endroit où les oiseaux ont dansé » après avoir obtenu leurs couleurs, qui apparait déjà sur des cartes du milieu du XvIII ${ }^{\mathrm{e}}$ siècle, ou encore les deux sauts Masala, (gouffre), éloignés de $35 \mathrm{~km}$ qui communiquent par un tunnel souterrain par où circulerait un anaconda géant (moyu wasu). On aura noté que la figure du serpent géant, faiseur de territoire et de culture est au cœur de cette représentation de l'espace. Tout ceci nous replonge dans la quasi-totalité des représentations amérindiennes de l'Amazonie. 
36 En parcourant l'Oyapock et ses affluents, c'est toute une géographie historique et mythique qui peut ainsi être esquissée. Les peuples vivant ou ayant vécu le long de ces cours d'eau ont inscrit leur histoire des origines à nos jours dans cet environnement qu'ils connaissent et parcourent depuis des siècles. L'important est que cette géographie mythique et magique demeure vivante dans la pensée des Wayãpi et des Teko qui en sont les dépositaires. Les Wayãpi ne racontent-ils pas que le cours rectiligne de l'Oyapock fut créé par le vol du martin-pêcheur (yawasi) ? Puis l'anaconda (moyu), en reptant tout doucement, en façonna les méandres que l'eau de pluie emplissait au fur et à mesure. Enfin, il revint au poisson goret (mili) (Lorcarriidae) d'en creuser les longs biefs (les « pointes » en créole).

\section{...aux épisodes historiques}

La toponymie témoigne également d'épisodes historiques ou anecdotiques récents, et la rivière Camopi en est tout particulièrement parsemée. Elle garde en mémoire la première ruée vers l'or datant de la fin du XIX ${ }^{\mathrm{e}}$ siècle et qui s'étiola au début de la Seconde guerre mondiale. À cette époque, cette rivière était continuellement parcourue par des Créoles et des Saramaka (Noirs Marrons venus du Surinam) et quelques aventuriers européens. Ainsi, on rencontre la crique Jalbot, du nom d'un orpailleur de cette époque, le dégrad Saint-Pierre, du nom d'un orpailleur venant de cette ville martiniquaise, ou bien le joli nom évocateur de « Roche Habillé des dames », belle roche plate (Takulu tape en teko) où les femmes s'arrêtaient pour se parer de leurs plus beaux atours avant d'aller danser au casino (salle de bal) du village d'orpailleurs de Camopi (situé bien plus en amont que l'actuel bourg amérindien). Ainsi, la mémoire des Teko contemporains est dépositaire de cette période aujourd'hui révolue, contée tout en nuances par leurs parents et grandsparents et inscrite dans les toponymes.

La toponymie actuelle, nous l'avons montré, garde aussi en mémoire des couches d'occupation territoriale bien plus anciennes, antérieures à l'arrivée des Wayãpi et des Teko dans la région, ce qui prouve à la fois leur fusion avec les anciens occupants de la région, et l'existence de relations politico-culturelles sur des territoires immenses. On retrouve des toponymes témoignant de l'activité missionnaire ancienne dans l'Oyapock au XVIII ${ }^{e}$ siècle : ainsi le lieu-dit contemporain de Saint-Soi est la déformation de NotreDame de Sainte-Foy, nom de la mission jésuite (1740-1763) dont l'implantation principale se situait au niveau de l'actuelle gendarmerie de Camopi, l'église se trouvant à l'emplacement du grand fromager du haut du bourg. Plus en aval, la « Roche Mon Père » a été ainsi nommée en souvenir du lieu de pique-nique des missionnaires à l'époque où ils parcouraient ce fleuve à la pagaie entre les missions de St-Paul et de Ste-Foy à la recherche d'âmes à convertir...

Sur l'Oyapock, le toponyme Keimukwale ("Antre de l'anaconda mythique » à qui les oiseaux prirent leurs couleurs), témoigne lui aussi d'une occupation amérindienne plus ancienne que celle des deux sociétés actuelles, de langue tupi-guarani. En effet, le mot keimu provient de la famille linguistique caribe (auquel appartiennent les groupes contemporains Kali'na et Wayana) : il dérive du terme kali'na okoyumo ou wayana okoyu imë; on peut penser qu'il est passé dans la langue wayãpi d'aujourd'hui par l'intermédiaire des Piriu ayant vécu sur l'Oyapock jusqu'au début du XIX ${ }^{e}$ siècle, peuple 
tupi-guarani qui parlait aussi le galibi véhiculaire (ancien nom du kali'na) dont l'usage avait été diffusé par les Pères Jésuites.

\section{Quelques exemples}

40 La présentation détaillée des toponymes wayãpi et teko nécessiterait un livre entier, tâche qui reste à concrétiser et dont les éléments sont pour l'essentiel rassemblés. Néanmoins la finalisation de ce travail devra mobiliser l'énergie des jeunes générations, certaines données étant politiquement sensibles. En particulier, nommer avec précision dans sa langue la géographie d'une région, permet ensuite d'appuyer des revendications territoriales. Nous avons choisi ici de présenter, d'aval en amont, quelques toponymes wayãpi et teko très remarquables avec leur histoire.

\section{Fleuve Oyapock}

41 - Wilatuluake (Roche canari zozo)

Ce toponyme cristallise à lui seul, les couches de peuplement qui se sont succédé dans la région depuis 250 ans. Jusque dans les années 1970 tous les habitants de l'Oyapock pouvaient livrer un discours sur ce nom de lieu. En créole, il signifie "Poterie de l'oiseau" et renvoie à son nom wayãpi, Wilatuluake, (Ancienne poterie des oiseaux). Son second nom est Wilakawawe, (Là où les oiseaux ont bu). Les Wayãpi racontent comment un couple de colibris en parade nuptiale réussit à tuer un anaconda géant, ce qui permit aux oiseaux de se peindre avec ses excréments irisés. Tous noirs dans les temps mythiques, les oiseaux doivent leurs magnifiques couleurs à cet anaconda fabuleux.

42 Après s'être peints, les oiseaux burent le cachiri contenu dans cette poterie, et la retournèrent une fois vidée. L'anaconda, maitre des couleurs, de l'arc-en-ciel et des motifs dessinés, est un grand classique de la pensée des peuples amazoniens.

Le nom Wilakawawe apparaît sur la carte d'Audiffredy de 1763, soit avant l'arrivée des Wayãpi en Guyane à partir de 1810. Sans doute faut-il voir là un héritage des Piriou (Piliyu), ancien peuple tupi aujourd'hui éteint ayant vécu dans cette zone avant eux.

Marquant la limite entre les communes de Saint-Georges de l'Oyapock et de Camopi sur la rive droite du fleuve, cette roche proéminente ovoïde, ressemblant à une jarre retournée, est connue sous le nom de Canari-zozo par tous les Oyapockois. À la fin du xxe siècle cette roche, jamais entièrement recouverte même en très hautes eaux, est aussi devenue la limite symbolique entre le territoire de pêche et de chasse des habitants de Saint-Georges et celui des habitants de Camopi.

45 - Wilapolaytawe

Ce lieu-dit n'est visible qu'à la saison sèche. En baissant, le niveau du fleuve découvre une grande île sableuse et plate.

Les Wayãpi nomment précisément ce lieu Wilapolaytawe (Ancienne aire de danse des oiseaux). Nouvellement parés des couleurs de l'anaconda, les oiseaux dansèrent sur le banc de sable avant de prendre leur envol pour leurs habitats respectifs.

- Roche Mon Père et Cette savane-roche, plongeant directement dans l'Oyapock du haut de ses 200 mètres, est nommée Taparap sur la carte d'Audiffredy de 1763. Elle est connue de tous les Oyapockois sous le nom créole de Roche Mon Père depuis le XIX ${ }^{e}$ siècle. Le nom actuel apparaît pour la première fois dans le rapport de Bodin en 1824. Elle était l'étape 
des missionnaires jésuites entre leurs missions de Sainte-Foy, à Camopi, et de Saint-Paul, près du confluent de la Notaye. On remarquera que c'est le terme créole qui est aujourd'hui adopté par les Teko et les Wayãpi.

47 - Yeloikeae (Case kalé)

Le nom du saut signifie «[le village] qui fut englouti» ou en créole "les maisons ont sombré ». Les Wayãpi et les Teko associent ce saut et surtout le remous profond bordé d'un amas rocheux qui le jouxte en aval à une belle légende. Existait là un village amérindien qui surplombait le saut. Un anaconda s'étant transformé en beau jeune homme avait séduit la fille du chef. Mais pendant ses ébats amoureux, il redevenait serpent et fut surpris par sa belle-mère qui fit tomber la résine de son flambeau dans son œil. C'est pour se venger qu'il engloutit le village. On dit qu'il vit désormais au fond de l'eau avec sa bien-aimée, seule survivante.

48 - Kumalawa

Ce saut important est mentionné sur la carte d'Audiffredy de 1763. Ce sont les ancêtres qui lui ont donné ce nom dont on ne connaît pas la signification. Dans ce cas, on dit qu'il s'agit de la « langue des esprits » (ayã aiwu).

49 - Meikolo yuka'e

C'est la passe principale du saut Kumalawa, (Là où les Noirs ont tué). Ce nom commémore l'assassinat, sur la grande roche de la rive gauche, du grand chef wayãpi Waninika par des Boni en 1842. Cet événement prend place dans une suite de tensions importantes dans la colonie. Fuyant les exactions des Ndjuka sur le Maroni, des Boni, traversant la Guyane vers l'est, tentèrent de s'implanter dans l'Oyapock. En 1841, eut lieu à Cafésoca le massacre par la troupe française d'un groupe de Boni cherchant à parlementer. Les Wayãpi, quant à eux, étaient peu désireux de devoir partager leur territoire. L'année suivante, des Boni en visite chez le chef suprême des Wayãpi, rejetèrent les rituels d'accueil (cachiri et repas collectifs) dans la crainte d'être empoisonnés.. Ils quittèrent en désordre le village wayãpi, poursuivis par un Waninika humilié. Abandonné par ses hommes, le chef les affronta seul et succomba sous leurs coups. Conscients de la gravité des faits, les Boni rebroussèrent chemin vers l'ouest. Ce fut leur dernière incursion sur l'Oyapock.

50 - Tayaunõlõnga

L'abbé Fournier mentionne cette montagne remarquable dès 1824. Il s'agit d'une "montagne couronnée », site de village amérindien anciennement fortifié. Son nom signifie «Là où les pécaris à lèvres blanches ont grogné ». Les Wayãpi et les Teko disent que le site était habité par les Kalanã, aujourd'hui éteints, Amérindiens petits mais agressifs comme des pécaris. Ils furent attaqués et vaincus par une expédition d'Amérindiens alliés à des Français. Cet événement conté par le chef wayãpi Norbert Suitman est attesté par les archives du début du XviII ${ }^{\mathrm{e}}$ siècle et donc bien antérieur à l'arrivée des Wayãpi et des Teko dans la région.

$51 \quad$ - Alikoto

Ce serait un esprit qui aurait donné son nom au saut. Il est déjà mentionné sur la carte d'Audiffredy de 1763 qui le décrit comme « long d'environ 800 pieds et à peu près 10 pieds de chutes ». Depuis 1830, de nombreux villages wayãpi ont existé en amont et en aval de ce saut qui est une pêcherie de première importance et possède des dégrads de roches plates particulièrement agréables pour les activités villageoises. 
52

Ce toponyme est mentionné sur la carte d'Audiffredy de 1763. Il s'applique à un saut et une grande île. C'est le nom d'un esprit anthropophage aquatique. Pour les Créoles guyanais, les maskililis sont des petits lutins forestiers qui persécutent les chasseurs. Le chef wayãpi Eugène Inãmu dirigea un grand village de 1955 à 1982 à l'extrémité amont de l'île.

53 - Alaliyo

Ce saut apparait sur la carte de Brodel et Mentelle de 1778. Ce serait un esprit qui aurait donné un nom au saut et au bassin situé en aval. Jadis, une grand-mère y était tranquillement en train de pêcher à l'aide du poison kunami. Un martin-pêcheur qui passait par là, jaloux des prises de la grand-mère, demanda à l'anaconda caché dans le bassin de l'effrayer. L'enfant qui l'accompagnait, comprenant le piège qui était en train de se mettre en place, essaya d'entraîner la grand-mère paralysée de peur par l'anaconda. Mais il était trop tard, la pirogue se retourna et les martins-pêcheurs purent récupérer le butin de la grand-mère.

54 - Mutusi itu

"Saut des moutouchis », car ces arbres sont très abondants sur les rives. Le nom de ce saut apparaît pour la première fois dans l'ouvrage de Jules Crevaux de 1883. Il fut le siège du grand village de Pierre Ka'iluwiyã, collaborateur de Henri Coudreau dans son exploration des affluents de l'Oyapock en 1889-90.

55 - Yengalali

« Rivière des chansons ». Le nom de cette crique apparaît en 1824 dans le travail de Bodin. Il y avait en amont un grand village où l'on trouvait de très bons chanteurs wayãpi. De fait, cette crique fut le siège de plusieurs villages wayãpi au début du XIX ${ }^{e}$ siècle.

56 - Yawapa

Littéralement «Jaguar anciennement ». Nom d'une crique du haut Oyapock. Les anciens ont vu là, sur les roches situées près du confluent, un énorme jaguar. C'est l'un des lieux où les Wayãpi, lors de leur migration vers le nord en provenance de l'actuel Amapá, se scindèrent en deux après que le tronc jeté en passerelle en travers de la rivière, étroite à cet endroit, se fut symboliquement brisé. Ceux qui n'avaient pas encore traversé, restant au Brésil, repartirent vers le sud, les autres s'enfoncèrent en Guyane.

Aujourd'hui y est installé le village du chef Laurent Pilauku.

57 - Itu wasu (Trois Sauts)

Le nom wayãpi du saut signifie «Saut géant ». Il est l'entrée de la région des sources de l'Oyapock. Sur sa rive gauche se trouve, juste en aval, le village Roger, le plus méridional de la commune de Camopi et même de la Guyane tout entière.

Son nom français actuel, qui n'apparaît qu'au milieu du XIX siècle, est dû à une mauvaise compréhension du nom wayãpi, Itu wasu devenant Trois Sauts par la ressemblance des sonorités. De fait, il ne comporte par trois niveaux mais quatre, faisant de celui-ci une barrière naturelle infranchissable par canot. Il est aussi l'antre d'un anaconda monstrueux, moyuu en wayãpi.

Ce saut a longtemps été une séparation physique isolant les groupes wayãpi du haut fleuve du monde du bas cours de l'Oyapock et du littoral. Les Wayãpi qui vivent actuellement à Zidock, Pina, Yawapa ou Roger sont tous originaires d'anciens villages situés en amont de ce saut. Ce n'est qu'à partir de 1969 que deux villages (Roger et Zidock) furent installés en aval du saut. 
- Mitake

"Vieille plateforme de chasse ". Le nom de cette crique apparaît au XIX ${ }^{e}$ siècle; auparavant elle était nommée Akao (aka'i, une espèce proche du cacao, Theobroma velutina). Les Wayãpi appellent cet endroit Mitake car de grandes pinotières s'y sont développées, et à la saison des fruits du palmier wassey, les toucans viennent s'en délecter. Du temps de la chasse à l'arc, les Amérindiens confectionnaient à cet endroit des affûts de chasse en hauteur (mita) afin de flécher aisément ces oiseaux.

\section{Rivière Camopi}

59 - Takulu tape (Roche habillé des dames)

« Roche plate ». À l'époque du village d'orpailleurs de Camopi (1925-1953) situé en amont de la Crique Alikene, les femmes des placers s'arrêtaient sur cette roche pour mettre leurs plus beaux atours avant de débarquer et d'aller danser.

60 - Dzawat a itu (Saut chien)

"Saut chien ». Ce saut, qui peut être difficile à franchir à la saison sèche, a été nommé ainsi en souvenir d'un accident de pirogue ayant causé la noyade d'un chien. Celui-ci a été enterré en tête de l'îlet du même nom. Précisons que les chiens sont des animaux très importants pour les Amérindiens. Les maltraiter peut entraîner l'errance de l'âme de son propriétaire après sa mort.

61 - Takulu tsĩng

« Roche blanche ». Ce rocher blanchâtre, se distinguant nettement des autres roches du secteur, se situe sur le moyen cours de la rivière Camopi à 2 heures de pirogue du bourg du même nom.

Comme beaucoup d'éléments remarquables de la nature, cette roche possède des pouvoirs singuliers. Les Teko disent que si l'on montre cette roche du doigt ou si l'on prononce son nom, il va se mettre à pleuvoir.

62 - Takulu palapi (Roches assiettes)

«Assiettes de pierre ». Ces roches disséminées sur plusieurs centaines de mètres possèdent la caractéristique d'être plates pour certaines d'entre elles. Les Teko les comparent à des assiettes, anciennement en terre cuite et façonnées par les femmes, aujourd'hui achetées dans le commerce.

63 - Takulu onaonan

Lieu-dit «Caillou qui court, qui court... ». Ce chaos rocheux situé sur la rive gauche de la Camopi proche de l'embouchure de la crique Tsitonõng tiãkã (crique Citron) constitue aux yeux des Teko un lieu magique qui ne doit pas être cité et où il vaut mieux éviter camper. Les roches y sont animées d'une vie propre. En effet, une famille teko y a déjà été attaquée par de nombreux êtres dangereux. Déjà, pendant la période de la première ruée vers l'or de la fin du XIX ${ }^{\mathrm{e}}$ siècle, un obiaman saramaka aurait travaillé à cet endroit...

64 - Floris uwa'a

«Bassin Floris ». Toponyme rappelant l'intense activité d'orpaillage et son cortège de violence lors de la première ruée vers l'or ayant duré jusque dans l'entre-deux guerres. Ce bassin a été nommé en souvenir de cadavres d'orpailleurs, dont un certain Floris, retrouvés à cet endroit. Ils avaient dérivé depuis le village Bienvenue situé en amont.

65 - Saut Yaniwe, Dzaliwe itu

Situé à une journée de pirogue du bourg, c'est le plus grand saut de la rivière Camopi. Il 
apparait déjà dans les textes du début du XviII ${ }^{e}$ siècle comme un obstacle considérable pour les Français qui allaient collecter le cacao à la source. Autrefois, racontent les Teko, un caïman géant habitait dans une de ses passes nommée Dzakalekwat (Trou du caïman). Il dévorait les hommes qui passaient par là. Aujourd'hui, il a été rendu inoffensif par un charme. On notera que le teko dzaliwe renvoie à aliwe qui désigne le caïman en wayana.

Il constitue la limite amont du terroir agricole des Teko actuels habitant cette rivière, leurs abattis et campements de culture s'étalant sur ses deux rives jusqu'à ce grand saut. $\mathrm{Au}$ delà, seules la chasse, la pêche et la cueillette sont pratiquées, on ne rencontre plus ni village ni abattis contemporains. Divers villages anciens des Teko ont existé dans cette région, le dernier ayant été abandonné dans les années 1960.

Situés en Zone de droits d'usage collectifs (ZDUC), ce saut et ses alentours sont un haut lieu de pêche et de chasse pour les Teko, dont c'est le cœur du territoire contemporain.

66 - Rivière Inipi, Ĩdipi

Cette rivière est déjà indiquée sous ce nom par les Pères Grillet et Béchamel en 1674, resté inchangé jusqu'à aujourd'hui. Les Teko ne le traduisent pas, bien qu'il ait été indiqué aux Européens par des populations parlant comme eux des langues tupi-guarani.

C'est par cet affluent que les premiers explorateurs, en provenance de l'Approuague, pénétrèrent dans la Camopi et de là dans l'Oyapock. Cette rivière devint surtout le principal axe de circulation des Teko dont les villages étaient alors dans le haut Inini. Les deux bassins étaient reliés par ce que l'on nomma plus tard «Ancien chemin des Émérillons ». L'actuel «Chemin des Émérillons » allant de la Tamouri à la Ouaqui n'a été utilisé qu'à partir des années 1920.

67 - Roche Bicyclette

Située sur la rivière Inipi, affluent de la rive gauche de la rivière Camopi, ces roches sont à plus d'une journée de pirogue du bourg.

Il est probable que le nom de Roche bicyclette ait été donné jadis par des orpailleurs créoles ou par des métropolitains, ces grands rochers plats dressés rappelant des roues de bicyclette. En effet, le Dr Heckenroth qui remonta la Camopi puis l'Inipi en septembre 1941 note déjà ce toponyme dans son carnet de route (Pourtal-sourieu 2012). Or cette zone est, depuis le premier cycle de l'or de la fin du XIX $x^{e}$ siècle et jusqu'à aujourd'hui, un lieu d'activité pour les orpailleurs.

Il est à noter que de nombreux toponymes créoles des rivières Camopi et Inipi constituent un témoignage unique de cette période de la première ruée vers l'or guyanaise. En utilisant ces toponymes créoles non traduits dans leur langue, les Teko demeurent les seuls à se souvenir de ces villages (Bienvenue, Tampak...) ou des noms d'orpailleurs (Jalbot, Cléna...), conservant ainsi la mémoire de ce pan oublié de l'histoire de la Guyane.

68 - Tipoko Dzawat (Roche crabe)

« [Roche] Crabe-jaguar ». Situé sur la rivière Inipi, cette roche remarquable est couverte de nombreux pétroglyphes. En plus des polissoirs couvrant sa partie sommitale, en saison sèche, on peut admirer de nombreux motifs anthropomorphes accompagnés de dessins semblant représenter des macaques à la queue enroulée ainsi que des amphibiens. Comme tous les pétroglyphes, il est impossible de les dater et donc très difficile de les attribuer à tel ou tel peuple, quant à comprendre leur rôle... Elle est nommée Roche jésuite ou Roche crabe sur les cartes depuis que le Dr Heckenroth remonta cette crique en 1941. Le nom teko Tipoko dzawat rappelle son origine magique. En effet, au XVIII ${ }^{\mathrm{e}}$ siècle, à l'époque où les Kali'na (appelés Taida par les Teko) remontaient jusque sur la rivière Inipi 
pour guerroyer, un chamane teko créa un monstre anthropophage pour protéger les siens. Celui-ci dévora les guerriers kali'na et, depuis, demeure pétrifié et inoffensif.

69 - Tsiminiwip talupawit

«Ancien village sous le courbaril ». Il a été fondé dans les années 1950 par la famille Civette, une importante et très ancienne famille teko ; il en existe deux emplacements : le premier, sur la rive droite, fut abandonné au profit du second, sur la rive gauche, après le décès de la fille du grand-père Civette. Ce site de la haute Camopi est en amont du saut Yaniwe près du saut du même nom (Tsiminiwip itu).

\section{Conclusion}

Toponymie historique, mythique ou anecdotique, appellation de zones de chasse, de pêche ou de cueillette, cette grande richesse dans la nomination montre une profonde connaissance de leurs lieux de vie par ces peuples ayant su, tout au long de leur histoire, apprivoiser et s'approprier cet univers d'eaux et de forêt mêlées. Les cartes que nous présentons ici restituent les toponymes tels qu'ils sont connus et énoncés par les populations actuelles de la commune de Camopi. Ils doivent être considérés comme l'aboutissement d'une démarche patrimoniale partagée par tous et bien ancrée dans la vie quotidienne des villageois. Cette toponymie est à la fois historique, confortant alors un lointain passé, et vivante, nommant les lieux les plus infimes de l'occupation intense contemporaine. Nous sommes donc en présence d'une double toponymie une première, plus héroïque, valant pour le temps long et une seconde pour le temps court, plus labile. Il y a gros à parier qu'il en fut de même autrefois...

71 Les cartes historiques que nous avons produites montrent d'ailleurs parfaitement que les Wayãpi et les Teko ont occupé avec une intensité variable la presque totalité du territoire que nous envisageons.

72 Soulignons à nouveau que le présent article qui reprend un carnet toponymique destiné aux habitants de Camopi (Grenand et al. 2015) est le résultat d'une recherche participative qui a permis de systématiser, de compléter et de localiser définitivement des données accumulées sur plus de quatre décennies. D'une certaine manière on peut affirmer que les cartes produites proviennent du savoir de la totalité des personnes adultes teko et wayãpi vivant ou ayant vécu entre 1970 et 2011.

Ce travail a pu être réalisé grâce au financement du Parc Amazonien de Guyane et de l'Observatoire Hommes-Milieux Oyapock (UMSR LEEISA) dans le cadre d'une aide de l'Etat gérée par l'Agence Nationale de la Recherche au titre du Labex DRIIHM, programme "Investissements d'avenir » portant la référence ANR-11-LABX-0010.

Nous remercions également la commune de Camopi ainsi que la collaboration active de tous ses habitants sans qui ce travail n'aurait pu être possible. 


\section{BIBLIOGRAPHIE}

Audiffrédy 1763 - Carte de la rivière d'Oyapock levée géométriquement et dessinée sur les lieux / présentée à Monseigneur le Duc de Choiseul ; par le Chevalier d'Audiffredy Offi. Réf. des anciennes troupes de la colonie de Cayenne, 1763. Cartes et Plans, Bibliothèque Nationale. Guyane $n^{\circ} 255$ (4 feuilles) et aussi Archives Nationales d'Outremer, Base Ulysse. Guyane n¹94, n $195, n^{\circ} 196$ et $n^{\circ} 197$.

Bagot Th. de 1849 - Rapport de M. Bagot sur les Indiens de l'Oyapock et jusqu'à l'Amazone, 8 mars 1849, Archives Emile Abonnenc.

Bodin E. 1824 - Mission Bodin aux sources de l'Oyapoc, ANOM/ Guyane E10 (10).

Coudreau H.A. 1893 - Chez nos Indiens : quatre années dans la Guyane Française, 1887-1891. Paris, Hachette, 609 p., 1 carte hors-texte.

Crevaux J. 1883 - Voyages dans l'Amérique du Sud. Paris, Hachette, 635 p.

Davy D. 2007 - Vanneries et vanniers. Approche ethnologique d'une activité artisanale en Guyane française . Thèse de doctorat en Ethnologie, Université d'Orléans, 527 p.

Davy D., Tritsch I. \& Grenand P. 2012 - Construction et restructuration territoriale chez les Wayãpi et Teko de la commune de Camopi, Guyane française. In Dossier Oyapock, Dossiê Oiapoque, Confins 16. URL : nhttp://confins.revues.org/7964 ; DOI : 10.4000/confins.796

Fleury M., Alupki T., Opoya A. \& Aloïke W. 2016 - Les Wayana de Guyane française sur les traces de leur histoire. Revue d'ethnoécologie 9.

Grenand F. 2009 - La langue Wayampi. In : Renault-Lescure O. \& Goury L. (Ed.) Langues de Guyane, Vent d'Ailleurs-Ird : 100-111.

Grenand P. 1982 - Ainsi parlait nos ancêtres : Essai d'ethnohistoire wayãpi. Paris, Éditions ORSTOM, $408 \mathrm{p}$.

Grenand P., Grenand F. \& Ouhoud-Renoux F. 2000 - Entre fleuve et forêt : stratégies adaptatives du peuplement wayãpi depuis le XVIII ${ }^{\mathrm{e}}$ siècle. In : Bahuchet S., Bley D., Pagezy H. \& Vernazza-Licht N. (Ed.) L'Homme et la forêt tropicale. Châteauneuf de Grasse, Éditions de Bergier : 223-235.

Grenand P., Moretti C., Jacquemin H. \& Prévost M.-F. 2004 - Pharmacopées traditionnelles en Guyane : Créoles, Wayãpi, Palikur, $2^{\mathrm{e}}$ édition entièrement revue et corrigée. Paris, IRD Éditions, 816 p.

Grenand P., Joubert P., Grenand F. \& Davy D. 2015 - Cartes et Toponymes des territoires Teko et Wayãpi, Parc Amazonien de Guyane. Cayenne, CNRS.

Heckenroth M. 1939 - Territoire de l'Inini. Rapport géographique - politique - médical et économique de la tournée effectuée du 27 juillet au 15 Septembre 1939 sur le bassin de l'Oyapock (cours moyen- supérieur-et les sources) par M.M. J. Baup, Adjoint des SC., Chef de la Circonscription de l'Oyapock, et M. Heckenroth, Médecin-lieutenant des TC., Poste de Cafésoca, 1939 [document révisé et complété en 1941].

Hurault J. \& Fribourg-Blanc A. 1949 - Mission astro-géodésique de l'Oyapock (Guyane Française), juillet à novembre 1947. Paris, Institut géographique national, $131 \mathrm{p}$. 
Laval P. 2016 - Captures estuariennes : Une ethnoécologie de la pêche sur le bas Oyapock Thése de Doctorat, Muséum national d'Histoire naturelle, Paris.

Maurel P. 2009 - La langue Teko In : Renault-Lescure O. \& Goury L. (Ed.) Langues de Guyane, Vent d'Ailleurs-Ird : 91-99.

Mentelle S. \& Brodel F. 1778 - Partie du fleuve Oyapock par Mr Brodel, 14 janvier 1778. Archives Nationales d'Outremer, Base Ulysse, Guyane ${ }^{\circ} 49$.

Navet E. 1985 - Les Émerillons. In : La question amérindienne en Guyane Française, Ethnies 1-2, Paris, SIF : 18-19.

Milthiade J. \& Loret P. 1823 - Voyage de J. Milthiade et P. Loret d'Oyapock aux Emerillons et à la rivière Inini en tournant les sources d'Approuague en août et septembre 1822, ANOM/ Guyane E10 (06)

Pourtal-Sourieu M. (Ed.) 2012 - Plumes amérindiennes : Don Dr Marcel Heckenroth. Marseille, GrandCourtrai, Édition Snoeck et Musées de Marseille, 126 p.

Thébault de la Monderie F. \& Charvet 1819 - Voyage dans la Rivière d'Oyapock par Thébault et Charvet, Octobre et Novembre 1819, ANOM/ Guyane 43, E10 (03).

\section{RÉSUMÉS}

Le présent travail est une version sensiblement modifiée d'un texte diffusé par le Parc amazonien de Guyane destiné aux communautés Wayãpi et Teko du bassin de l'Oyapock et de la Camopi et secondairement aux écoles de la région (Grenand et al. 2015). Il analyse successivement l'histoire de la cartographie du territoire teko et wayãpi, la construction de la toponymie autochtone, pour terminer par la présentation d'une sélection de toponymes mettant en valeur tout à la fois la mémorisation des noms et la visualisation des lieux.

This work is a sensibly modified version of the text published by the Guianese Amazonian Park for Wayãpi and Teko communities of the Oyapock and Camopi regions and for this region's schools (Grenand et al. 2015). It is an analysis of the history and cartography of the Teko and Wayãpi territories, of the construction of natives' toponymy, and finally a presentation selected toponyms highlightin both name memorization and location visualization.

\section{INDEX}

Population Teko, Wayãpi

Keywords : toponymy

Mots-clés : toponymie

Index géographique : Guyane française, Oyapock, Camopi

\section{AUTEURS}

\section{PIERRE GRENAND}

Directeur de recherche émérite, IRD 
FRANÇOISE GRENAND

Anthropologue, Directeur de recherche émérite, CNRS, Membre du comité de direction de l'Observatoire Hommes-Milieux "Oyapock" du CNRS

\section{PIERRE JOUBERT}

Ingénieur cartographe, PAG

\section{DAMIEN DAVY}

Ingénieur de recherche, OHM Oyapock, UMSR LEEISA (CNRS-UG-Ifremer) 